\title{
Characterization of the droplet temperature in sprays using laser-induced fluorescence and time-correlated single-photon counting
}

\author{
Yangpeng Liu ${ }^{1,2}$, Stiti Mehdi ${ }^{1}$, Castanet Guillaume* ${ }^{* 1}$, Chaynes Hadrien ${ }^{1}$, Lemoine Fabrice ${ }^{1}$, \\ Xishi Wang ${ }^{2}$ \\ ${ }^{1}$ Université de Lorraine, CNRS, LEMTA, F-54000, Nancy, France \\ ${ }^{2}$ State Key Lab. Of Fire Science, University of Science \& technology of China, Hefei 230026, \\ P.R. China
}

*Corresponding author email: guillaume.castanet@univ-lorraine.fr

\begin{abstract}
The objective is here to develop a measurement technique based on laser-induced fluorescence lifetime (LIF). Measuring the fluorescence lifetime is a promising alternative to conventional approaches based on the intensity of the fluorescence signal. Given that the fluorescence lifetime is inversely proportional to the fluorescence quantum yield, it is dependent on temperature in the same manner as the intensity of the fluorescence signal. Intensity-based fluorescence do not yield absolute quantities and always require a reference to determine the temperature. Moreover, they are made complicated by the the fluorescence signal from a droplet which varies with many parameters in addition to the temperature such as the droplet size, the droplet position with respect to the laser beam and detector, the droplet shape. In contrast, measuring the lifetime by a technique either in the time-domain or in the frequency-domain provides directly an absolute measurement. In this study, the timecorrelated single-photon counting (TCSPC) is applied for measuring the temperature in water sprays. The study of the evolution of the fluorescence lifetime of kiton Red allow us to determine a good solution to obtain the better accuracy in temperature.
\end{abstract}

Keywords: Laser Induced fluorescence, Spray, Cooling

\section{Introduction}

The characterization of the droplet temperature in polydisperse sprays is an important challenge in many engineering applications. While the size and velocity of droplets can be measured using relatively widespread and well-established techniques, characterizing the temperature remains quite challenging (Lemoine and Castanet 2013). Laser-induced fluorescence (LIF) offers unprecedent temperature sensitivities. Examples of application include droplets spreading onto solid surfaces (Chaze et al. 2017), mono and multi-component fuel droplets combusting and evaporating in high-temperature environments (Perrin et al. 2015), supercooled droplets in icing processes (Stiti et al. 2019). Usually, an organic dye is dissolved into the liquid prior to its injection. The droplets are illuminated by a laser beam with an appropriate wavelength to allow light absorption by the fluorescent molecules which emit in return a fluorescence signal depending on the temperature by some of its properties (intensity, wavelength, lifetime). A few dyes, such as rhodamine B or kiton red (KR), have been used so far for temperature measurement in water solutions. Besides a high dependency of their fluorescence signal on temperature, these dyes have remarkably high absorption cross sections and high quantum fluorescence yields, which is advantageous for the implementation of the measurements.

Measurements are usually based on the intensity of the fluorescence signal. However, the intensity does not provide an absolute quantity and in spray, the fluorescence intensity from a droplet depends on many parameters in addition to the liquid temperature such as the droplet size, the droplet position with respect to the laser beam and the detector, the concentration in dye for evaporating droplets. As a result, it is generally unavoidable to use intensity ratios i.e., to measure the intensities of the fluorescence signal in two spectral bands which are divided by each other. However, this approach can suffer from several sources of error as reported 
(Labergue et al. 2010, 2012) due to the polydispersion in droplet size and multiple light scattering by the droplets which induces a significant out-of-field fluorescence.

As an alternative, measuring the fluorescence lifetime provides a direct means to access to an intrinsic parameter that is related to the key mechanisms of the fluorescence cycle, namely the fluorescence lifetime. The latter is closely dependent on the quenching rate of the fluorescence by collision with solvent molecules and therefore on the temperature. Contrary the fluorescence intensity, the lifetime value is not dependant on the measurement method and system,. Measurements will require a single dye and a single spectral detection band. The aim of the present study is to develop a very first application of TCSPC to measure the temperature of water droplets in a polydisperse spray.

\section{The effect of temperature on the fluorescence lifetime}

Fluorescence results from the spontaneous emission of a photon by molecules which has been excited to an electronic state of higher energy. In the case of laser induced fluorescence, the excitation comes from the absorption of a laser beam. Fluorescence typically follows firstorder kinetics. After an excitation by an ultrashort pulse of light, the fluorescence signal $I_{f}(t)$ emitted by the population of molecules will ideally decay with time according to:

$$
I_{f}(t) / I_{f}(0)=\mathrm{e}^{-t / \tau}
$$

where $\tau$ is the fluorescence lifetime. However, there are quite a few instances in the literature where biexponential decays were observed including rhodamine B (Kristoffersen et al. 2014). In this case, the decay be expressed by:

$$
I_{f}(t) / I_{f}(0)=a \mathrm{e}^{-\frac{t}{\tau_{1}}}+(1-a) \mathrm{e}^{-\frac{t}{\tau_{2}}}
$$

where $a$ is a parameter between 0 and 1 and an average fluorescence lifetime $\tau$ can be computed as (Casadevall I Solvas et al. 2010) as followed:

$$
\tau=a \tau_{1}+(1-a) \tau_{2}
$$

In the following, the fluorescence lifetime will be fitted by a biexponential decays. An Arrhenius law is widely accepted to describe the effect of temperature on the rate of the non-radiative deactivations for KR in many solvents (Mendels et al. 2008). For simplicity, this law was also considered for the temperature dependence of the fluorescence intensity and the lifetime (Mercadé-Prieto et al. 2017):

$$
\tau=A \exp \left(\frac{E_{a}}{R T}\right),
$$

where $E_{a}$ is the activation energy and $R$ is the gas constant $\left(R=8.314 \mathrm{~J} . \mathrm{K}^{-1} \cdot \mathrm{mol}^{-1}\right)$. For $\mathrm{KR}$, values of $E_{a}$ have been reported by (Mercadé-Prieto et al. 2017) based on their own measurements and data taken from the literature., Mendels et al. (2008) obtained a value of $E_{a}=24.2 \mathrm{~kJ} / \mathrm{mol}$. While the effect of the solvent and the chemical environment have been examined in several studies (Zhang et al. 2014; Hilaire et al. 2017), little consideration has been given to the dependence of $E_{a}$ on the emission wavelength. However, this dependence is strong enough to make it possible to use $\mathrm{KR}$ to measure the temperature with the two-color one-dye method (Lemoine and Castanet 2013).

\section{Experimental setup}

TCSPC is a method of choice for measuring the fluorescence lifetime in the time domain with an ultrashort pulsed laser. The technique is well established and is usually consider outperforming other lifetime measurement techniques in sensitivity, dynamic range and time resolution. Indeed, the elapsed time between the sample excitation by an ultrashort pulsed light source and the emission of a fluorescence photon can be measured typically with a picosecond accuracy. Figure 1 shows the optical system used for the implementation of the TCSPC technique. The excitation of the fluorescent dyes is performed by a femtosecond Ti:Sapphire laser (Coherent Chameleon Ultra II) which is tuneable from 690 to $1080 \mathrm{~nm}$ and generates light pulses with a width of about $140 \mathrm{fs}$. To induce the fluorescence of KR, the laser is firstly tuned on a wavelength of $980 \mathrm{~nm}$. Then, frequency of the laser beam is doubled using the second harmonic generator HarmoniXX from A.P.E Angewandte Physik \& Elektronik 
$\mathrm{GmbH}$. The resulting wavelength cantered around $490 \mathrm{~nm}$ allows obtaining an intense fluorescence signal with RhB.

a)

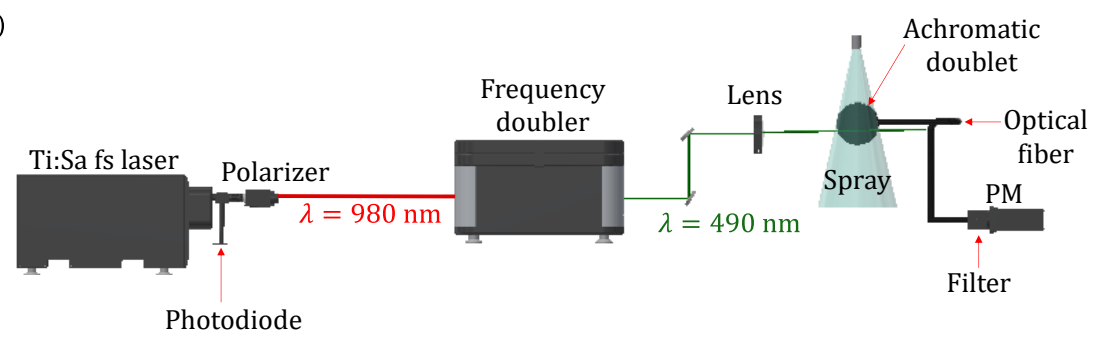

b)

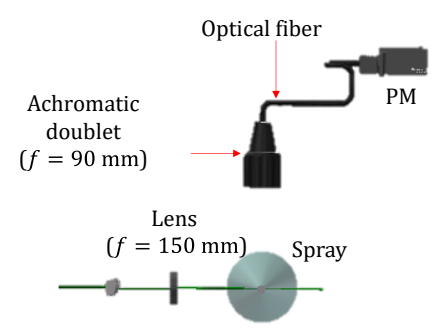

c)

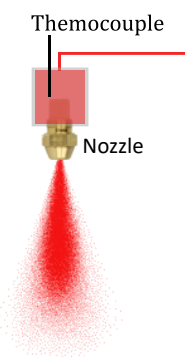

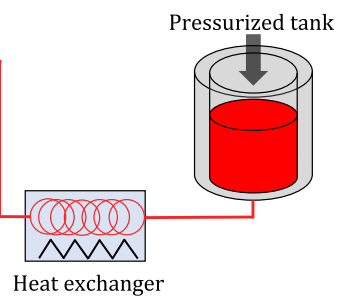

Figure 1: Experimental setup (a: Front view, b: Top view of the spray and the detection system, c: spraying system).

When the measurements are performed in the spray, the laser beam is focused by means of a lens with a focal length $f=200 \mathrm{~mm}$, allowing to obtain a beam waist diameter of about 100 $\mu \mathrm{m}$ and a Rayleigh length of about $6 \mathrm{~cm}$. A fast hybrid photomultiplier tube (R10467, Hamamatsu) with a Peltier cooler to reduce the dark count rate, is used for photon counting. The output of the detector is sent to a time-correlating counter, the HydraHarp 400 controller (PicoQuant $\mathrm{GmbH}$ ) which is operating in forward start stop mode to measure the arrival times of single photons. In short, a laser pulse causes a photon event at the detector and the electronics measures the delay between the laser pulse and the subsequent photon event. This measurement implies a sharp timing of the laser pulse, which is obtained from a fast photodiode (TDA 200 from PicoQuant $\mathrm{GmbH}$ ) also connected to the timing electronics. This photodiode allows detecting the laser pulses with a small-time jitter due a very sharp falling edge (Fall time typ. $250 \mathrm{ps)}$ ). It works on the light component in reflection from a beam splitter $(99 \% \mathrm{~T}, 1 \% \mathrm{R})$ placed at the laser output. The fluorescence signal is collected at right angle from the laser excitation using an achromatic doublet with a focal of $90 \mathrm{~mm}$ and the signal is transmitted by a single-mode optical fibre with a core diameter of $70 \mu \mathrm{m}$. Finally, different bandpass filters can be mounted in front of the photomultiplier tube in order to detect the photons on specific spectral bands.

The basic principles of TCSPC have been discussed in great detail in literature (Lakowicz 2013). The measurement method is based on the repetitive and precisely timed registration of single photons using the previously described system. By counting a high number of photon events to achieve statistical convergence, a histogram of the photon arrival times distribution can be built up. The width of the time bins in this histogram depends on the required time resolution (typically a few tens of ps). Finally,the fluorescence decay in the histogram is fitted by a biexponential function. A non-linear least squares analysis is used to test if the model is consistent with the data and estimate the parameters of the fluorescence decay model. The noise coming from the PM and the environment is also considered by adding "noise" photons uniformly distributed in time. Typically, in the dark, the count rate of the noise is about $60 \mathrm{Cps}$. 
a)

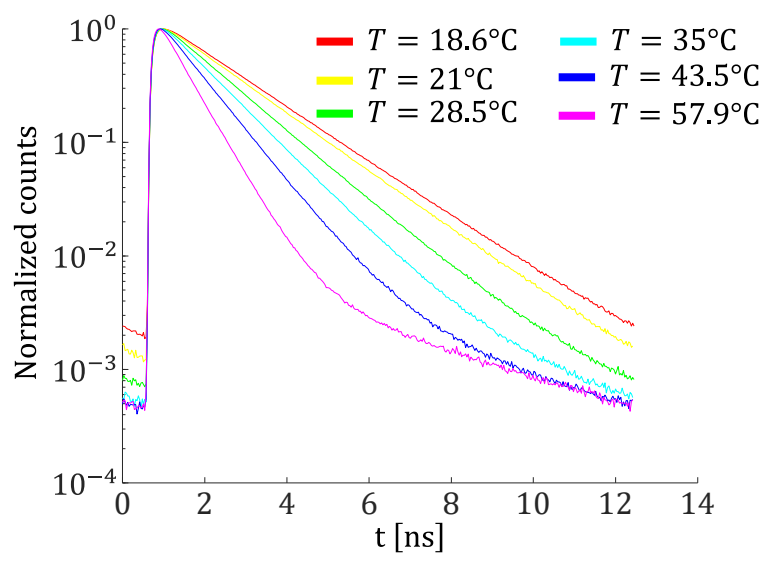

b)

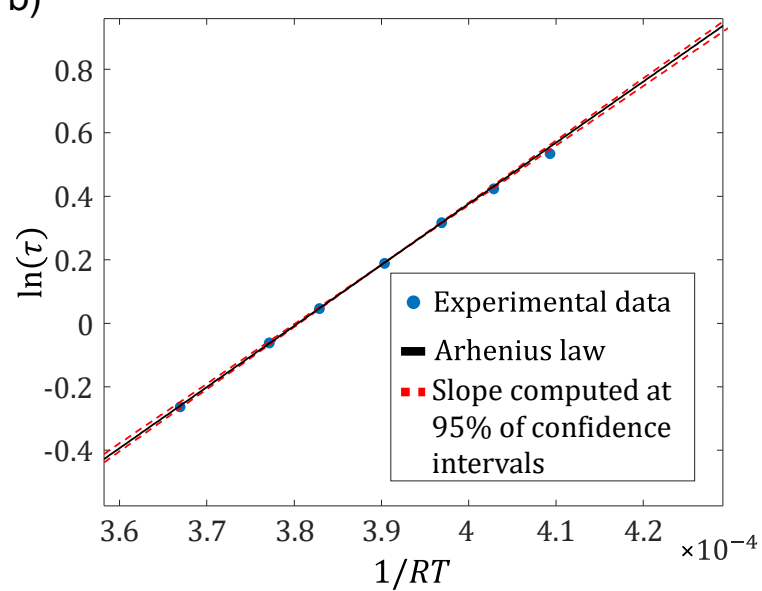

Figure 2: a) fluorescent decays of $\mathrm{KR}$ at different temperatures for the spectral bands [630 $\mathrm{nm}-$ $650 \mathrm{~nm}$ ]. Measurements carried out in a cell at a concentration of $10^{-5} \mathrm{M}$. b) fitting of a linear regression of $\ln (\tau)$ versus the inverse temperature to obtain the parameters of the Arrhenius equation $\left(\mathrm{E}_{\mathrm{a}}=18.7 \pm 0.4 \mathrm{~kJ} / \mathrm{mol}, \mathrm{A}=6.69 \pm 0.85\right)$.

\section{Temperature calibration}

Before measuring the droplet temperature in sprays, it is necessary to perform a lifetime temperature calibration. This step consists in measuring the fluorescence lifetime in a cell for different liquid temperatures. The liquid is stirred to ensure a uniform temperature while a thermocouple is positioned a few $\mathrm{mm}$ from the LIF measurement volume. These calibration experiments were carried out using a filter on the spectral band [630 nm - $650 \mathrm{~nm}$ ]. Figure 2 shows the fluorescent decay of KR at different temperatures. As observed the fluorescence lifetime is strongly dependant on the temperature. In Figure 2.b, $\ln (\tau)$ is plotted as a function of $1 / R T$ for $\mathrm{KR}$ in the band [630 nm $-650 \mathrm{~nm}$ ] and the linearity of the plot confirms the good suitability of the Arrhenus law (Eq. 4). The temperature dependence of $\tau$ is controlled by the activation energy $E_{a}$ which can be determined from the slope of this curve. Presently, an activation energy of $E_{a}=18.7 \pm 0.4 \mathrm{~kJ} / \mathrm{mol}$ is found

\section{Application to droplets in polydisperse spray}

To test the measurement method, a spray configuration which has been already examined in earlier studies (Labergue et al. 2010, 2012; Rimbert and Castanet 2010) was chosen, as it illustrates the problems encountered in measuring the temperature in polydisperse sprays with the intensity of the fluorescence signal and the two-color laser-induced fluorescence technique. The spray is generated by a pressure swirled atomizer (Danfoss oil nozzle, angle $30^{\circ}$ type S, $0.50 \mathrm{USgal} / \mathrm{h}, 1.87 \mathrm{~kg} / \mathrm{h}$ ) designed to generate a full cone spray. Before entering the nozzle, the liquid flows across a heat exchanger, which allows to control the liquid temperature during the injection. A type $\mathrm{K}$ thermocouple inserted in the inlet pipe of the spray nozzle is used to determine the liquid injection temperature. The spraying system is also mounted on translations stages to move the spray relatively to the optics and perform measurements at different positions. The size and velocity distributions of the droplets can be found in the study by Labergue et al. (2012). An increase of the injection pressure from 2 bar to 6.5 bar, induces a decrease in the statistical droplet diameter $D_{10}$ from $40 \mu \mathrm{m}$ to 20 $\mu \mathrm{m}$, while the droplet density increases from $10^{3}$ to $5.10^{4}$ particles per $\mathrm{cm}^{3}$ at $z=13 \mathrm{~mm}$ downstream of the injector outlet (Figures 5 and 6 in Labergue et al. 2012). 
a)

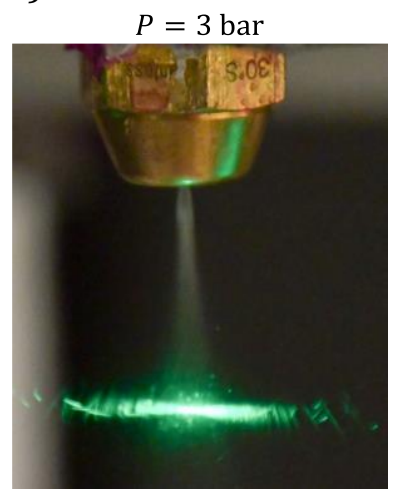

b)

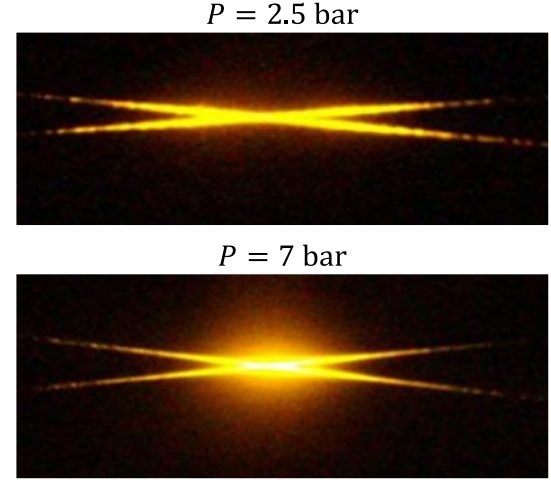

Figure 3: Images of the spray at different injection pressures, a: Illumination with a focalized laser beam at $490 \mathrm{~nm}$, b: Fluorescence field induced by two laser beams crossing each other in a configuration of Laser Doppler Velocimetry. Images extracted from Labergue et al. (2012). Photographs of the spray are showing evidence of multiple light scattering by the droplets.

Using the same spraying system, Labergue et al. (2010) found that a significant distortion of the fluorescence spectrum could be observed when changing the injection pressure and thus the droplet size (Figure 6 in Labergue et al. 2010). They described for the first time an effect apparently related to the size of the droplets, which is capable to alter the fluorescence ratio and thus to induce a bias in the droplet temperature measurements. Later, Labergue et al. (2012) have highlighted the importance of reducing the depth of field of the detection optics. In a spray, multiple light scattering by the droplets can be significant, especially when the injection pressure and thus the density of the droplets is increased. While the laser beam has typically a diameter of a few hundred microns in their measurements, light scattering considerably enlarges the region of the fluorescence excitation. Droplets normally situated well outside the laser beams emit a substantial amount of parasitic fluorescence (Figure 3b). Although most of this fluorescence originates from regions outside of the focal plane of the collection optics, effects on the intensity of the fluorescence signal can be significant. The detection system collects light originating from a region that has a significant depth on both sides of the focal plane (typically several $\mathrm{mm}$ ). In the two-color one-dye measurements method, the signal intensities detected on two emission bands of a single dye are used to calculate an intensity ratio $R$ and determine the temperature, provided two bands of different activation energies:

$$
R=I_{f_{1}} / I_{f_{2}}=\phi_{1} / \phi_{2}=\exp \left(\frac{E_{a_{1}}-E_{a_{2}}}{R T}\right)
$$

For KR on the spectral bands [535 nm - $545 \mathrm{~nm}$ ] and [615 nm - $750 \mathrm{~nm}$ ], a temperature sensitivity of the ratio of about $0.9 \% /{ }^{\circ} \mathrm{C}$ was reported by Labergue et al. (2012). However, the ratio is changing considerably when the injection pressure is increased from 3 bar to 6.5 bar (Figure 4a) while the spraying conditions are isothermal. The variations of the ratio $R$ are reduced with the long-distance microscope which removes more efficiently the out-of-field fluorescence (Figure 13 in Labergue et al. 2012). Nevertheless, converted into temperature, the remaining variations of $R$ still corresponds to more than $10^{\circ} \mathrm{C}$. The front lens used for collecting the signal in the present study has the same deficiencies. From measurements performed with a spectrometer, the ratio $R$ varies within a significant range when using the same spectral bands (Figure 4a). Labergue et al. (2012) developed an empirical method of correction using a third spectral band of detection. Although many measurement biases seem to be effectively corrected in their study, it cannot be ensured that all are completely removed due to the lack of a theoretical basis for these corrections.

Preliminary tests have been performed to verify that lifetime-based measurements are not affected by the problems discussed above. The injection pressure was increased from 2 bar to 6 bar while the injection temperature was fixed at $20^{\circ} \mathrm{C}$. Then, lifetime measurements were performed at $z=13 \mathrm{~mm}$, which is the same downstream position as the measurements 
shown in Figure 4.a. The results were then compared to measurements performed at the same temperature in a cell and in a monodisperse droplet stream where the droplet size is about $150 \mu \mathrm{m}$. Results, presented for $T_{i n j}=20^{\circ} \mathrm{C}$ in Figure 4.b, reveal that the fluorescent decay does not change significantly in all these situations. A lifetime difference of about 0.015 ns is observed. Such a small difference, corresponding to a bit more than $1^{\circ} \mathrm{C}$, can be partially explained by a deviation in the liquid temperature, despite much care has been taken to have the same temperature in the spray, the droplet stream and the cell. Finally, the main difference between the cell, and the spray appears to be in the noise contribution, which is slightly higher for the spray measurements. It obviously takes a longer time to acquire as many photons in a spray as in a cell due to a lower liquid volume fraction. However, as long as the noise level is known, the noise contribution can be taken into account in the fitting model. From these preliminary tests, it seems that no bias is affecting the temperature measurements using the TCSPC technique, as opposed to intensity-based measurements using the 2cLIF method.

a)

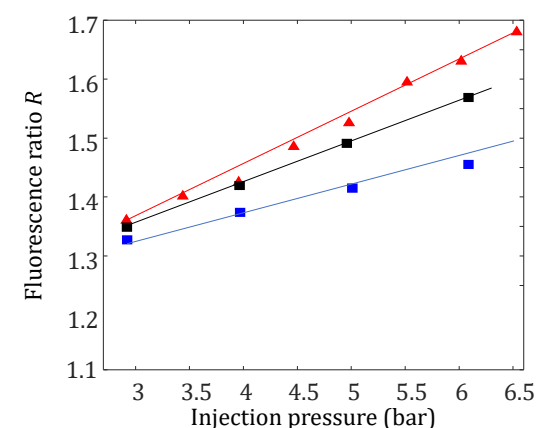

- Achromatic doublet $f=90 \mathrm{~mm}$ (present study)

¿- Achromatic doublet $f=310 \mathrm{~mm}$ (Labergue et al. 2012)

- Long distance microscope (Labergue et al. 2012) b)

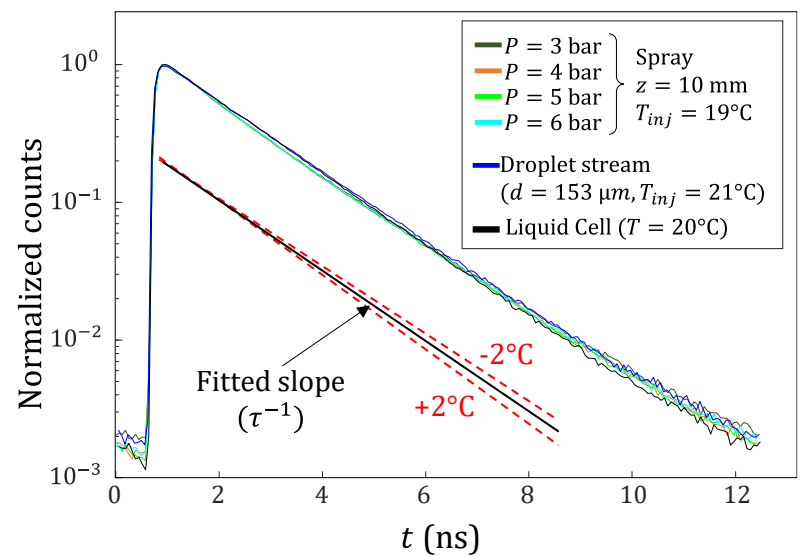

Figure 4: a) Evolution of the fluorescence ratio $R$ as a function of the injection pressure for different detection optics. The fluorescence signal is detected on the spectral bands [535 nm- $545 \mathrm{~nm}$ ] and $[615 \mathrm{~nm}-675 \mathrm{~nm}]$ downstream of the nozzle outlet at $z=13 \mathrm{~mm}$. b) fluorescent decay of KR in a spray ( $z=13 \mathrm{~mm}, P=5 \mathrm{bar})$, in a droplet stream $(\mathrm{at} z=10 \mathrm{~mm})$ and in a cell for a liquid temperature of $20^{\circ} \mathrm{C}$.

\section{Application to droplets in polydisperse spray}

The measurement technique was used to characterize the droplet cooling for different injection temperatures and an injection pressure of $P=5$ bar. Figure 5 presents the evolution of the droplet temperature on the spray axis for $T_{i n j}=19^{\circ} \mathrm{C}, 44^{\circ} \mathrm{C}$ and $60^{\circ} \mathrm{C}$. Due to evaporation, droplets injected at room temperature can be cooling down. While the cooling rate is dependent on the size, velocity and initial temperature of the droplets, the final temperature of the droplet depends basically on the ambient air temperature $T_{a m b}$ and the relative humidity $R H$. It corresponds to the wet-bulb temperature $T_{w b}$. Under the experimental conditions in Figure 5, $T_{a m b}=19^{\circ} \mathrm{C}$ and $R H=52 \%$, the wet bulb temperature can be valuated at $T_{w b}=13.2^{\circ} \mathrm{C}$ (Stull 2011). In Figure 5, measurements seem to converge towards this theoretical limit for the case $T_{i n j}=19^{\circ} \mathrm{C}$. For $T_{i n j}=44^{\circ} \mathrm{C}$ and $60^{\circ} \mathrm{C}$, the droplets have not reached their thermal equilibrium at $z=100 \mathrm{~mm}$, but their temperature is well below the ambient temperature. It should be mentioned that the spray was injected into a large room in which air is constantly refreshed. However, local humidity inside the spray is surely higher than in the ambient.

A comparison is also possible with measurements carried out by Labergue et al. (2012) using the intensity of the fluorescence signal. Measurements obtained by Labergue et al. (2012) for $T_{i n j}=51^{\circ} \mathrm{C}$ and $P=4.5$ bar have been added in Figure 5. An important difference can be observed between both measurement techniques. It is clear that the cooling rate is lower and does not follow the same time evolution. This can hardly be explained by differences in the 
injection conditions ( $P=4.5$ bar against $P=5$ bar), nor by the ambient conditions (unfortunately, Labergue et al. (2012) provide no information about the relative humidity or about the ambient temperature). More likely, intrinsic biases are affecting the intensity-based measurements performed by Labergue et al. (2012). The fluorescence signal is detected by means of two photomultiplier tubes and a threshold level is fixed significantly above the noise level of the channels of detection. If a sequence has more than ten consecutive samples above this threshold, it is considered coming from droplets otherwise the sequence is fully rejected. Obviously, this condition is more difficult to fulfill for very small droplets. The fluorescence signal emitted by a droplet varies roughly with $D^{3}, D$ being the droplet diameter. However, in intensity-based measurements, the dynamic range of the PMT detectors (ratio of the maximum signal and the noise level) is hardly greater than 15-20, making it difficult to study a droplet population whose size ranges over more than a 2.5:1 ratio. Considering that the high voltages of the PMTs were adjusted so as to detect the largest droplets without any saturation of the signal level, many of the small droplets could simply not be detected. This issue is not encountered in the case of the current lifetime-based measurement technique which operates on single photons with no distinction whether they originate from a small or a large droplet. Therefore, we believe that the temperature measured using TCSPC is very close to the volume average temperature of the liquid phase without any effect of the droplet size distribution of the spray.

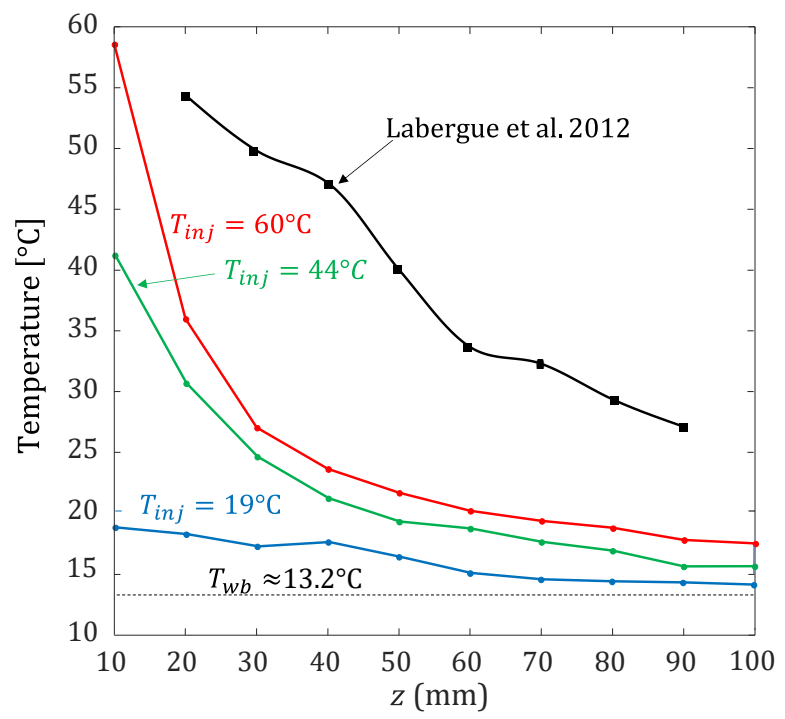

Figure 5: Evolution of the droplet temperature measured in the centreline of the spray for different injection temperature ( $P=5$ bar). Comparison to the data from Labergue et al. (2012) at $P=4.5$ bar.

\section{Conclusions}

A new method for measuring the droplets temperature in sprays was developed and tested. The method is based on the measurement of the fluorescence lifetime of certain dyes that are particularly sensitive to temperature such as kiton red. One of the major advantages of using the fluorescence lifetime is the fact that it is an absolute measurement, unlike the intensity, which is always relative. The fluorescence lifetime is an intrinsic molecular property, which is not affected by fluctuations in excitation source intensity or in the collections of the fluorescence signal. This makes all the difference when measuring the temperature in dense sprays. Although the technique is not suitable for measuring the temperature of individual droplets as opposed to intensity-based measurements (Labergue et al. 2012), it is possible to obtain the mean volume temperature of the liquid phase with no bias. Intensity-based measurements are usually limited by the dynamic range of their detectors, which are not able to detect with the same settings (gain voltage, laser power...) individual droplets when they have very different size. This problem is not encountered with lifetime measurements and the TCSPC technique which operates on single photons regardless of their origin (large or small droplets). 
A very high signal-to-noise ratio could be achieved under all the conditions which were tested in sprays. A dynamic range of about 3 decades for the decay curve can be retained in the spray, which is just slightly lower than in a liquid cell. This provides to the measurements, an accuracy and robustness which is probably unrivalled among the other temperature measurement techniques available in sprays. The absolute error typically does not exceed $\pm 1^{\circ} \mathrm{C}$ in most cases and even reduces to about $\pm 0.5^{\circ} \mathrm{C}$ if any uncertainty affecting the calibration coefficients are put aside.

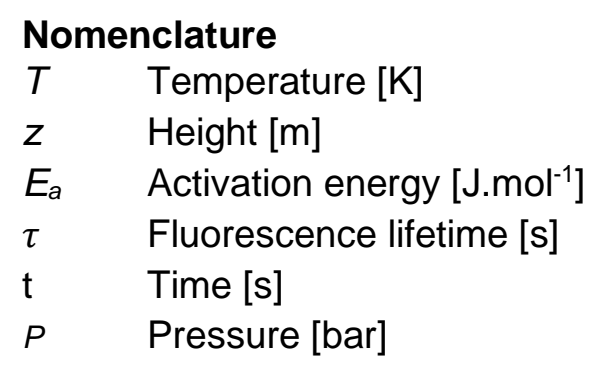

\section{References}

Casadevall I Solvas X, Srisa-Art M, Demello AJ, Edel JB (2010) Mapping of fluidic mixing in microdroplets with $1 \mu \mathrm{s}$ time resolution using fluorescence lifetime imaging. Anal Chem 82:3950-3956

Chaze W, Caballina O, Castanet G, Lemoine F (2017) Spatially and temporally resolved measurements of the temperature inside droplets impinging on a hot solid surface. Exp Fluids 58:

Hilaire MR, Mukherjee D, Troxler T, Gai F (2017) Solvent dependence of cyanoindole fluorescence lifetime. Chem Phys Lett 685:133-138

Kristoffersen AS, Erga SR, Hamre B, Frette $\varnothing$ (2014) Testing fluorescence lifetime standards using two-photon excitation and time-domain instrumentation: Rhodamine B, coumarin 6 and lucifer yellow. J Fluoresc. https://doi.org/10.1007/s10895-014-1368-1

Labergue A, Delconte A, Castanet G, Lemoine F (2012) Study of the droplet size effect coupled with the laser light scattering in sprays for two-color LIF thermometry measurements. Exp Fluids 52:1121-1132

Labergue A, Deprédurand V, Delconte A, et al (2010) New insight into two-color LIF thermometry applied to temperature measurements of droplets. Exp Fluids 49:547-556

Lakowicz JR (2013) Principles of fluorescence spectroscopy. Springer science \& business media

Lemoine F, Castanet G (2013) Temperature and chemical composition of droplets by optical measurement techniques: a state-of-the-art review. Exp Fluids 54:1572

Mendels DA, Graham EM, Magennis SW, et al (2008) Quantitative comparison of thermal and solutal transport in a T-mixer by FLIM and CFD. Microfluid Nanofluidics. https://doi.org/10.1007/s10404008-0269-5

Mercadé-Prieto R, Rodriguez-Rivera L, Chen XD (2017) Fluorescence lifetime of Rhodamine B in aqueous solutions of polysaccharides and proteins as a function of viscosity and temperature. Photochem Photobiol Sci. https://doi.org/10.1039/c7pp00330g

Müller CB, Weiß K, Loman A, et al (2009) Remote temperature measurements in femto-liter volumes using dual-focus-Fluorescence Correlation Spectroscopy. Lab Chip. https://doi.org/10.1039/b807910b

Paviolo C, Clayton AHA, Mcarthur SL, Stoddart PR (2013) Temperature measurement in the microscopic regime: A comparison between fluorescence lifetime- and intensity-based methods. J Microsc. https://doi.org/10.1111/jmi.12033

Perrin L, Castanet G, Lemoine F (2015) Characterization of the evaporation of interacting droplets using combined optical techniques. Exp Fluids 56:

Rimbert N, Castanet G (2010) Liquid Atomization out of a Full Cone Pressure Swirl Nozzle. 7th Int Conf Multiph Flow ICMF 2010 Tampa FL May 30 June 42010

Stiti M, Labergue A, Lemoine F, et al (2019) Temperature measurement and state determination of supercooled droplets using laser-induced fluorescence. Exp Fluids 60:1-13

Stull R (2011) Wet-bulb temperature from relative humidity and air temperature. J Appl Meteorol Climatol 50:2267-2269

Zhang XF, Zhang Y, Liu L (2014) Fluorescence lifetimes and quantum yields of ten rhodamine derivatives: Structural effect on emission mechanism in different solvents. J Lumin 145:448-453 